\title{
Transmission Electron Microscope Sampling Method for Three-Dimensional Structure Analysis of Two-Dimensional Soft Materials
}

\author{
Sang-Gil Lee ${ }^{\dagger}$, Ji-Hyun Lee ${ }^{\dagger}$, Seung Jo Yoo, Suvo Jit Datta ${ }^{1}$, \\ In-Chul Hwang ${ }^{1}$, Kyung-Byung Yoon ${ }^{1, *}$, Jin-Gyu Kim*
}

Nano-Bio Electron Microscopy Research Group, Korea Basic Science Institute, Daejeon 34133, Korea

${ }^{1}$ Center for Nanomaterials, Departments of Chemistry, Sogang University, Seoul 04107, Korea

\author{
${ }^{\dagger}$ These authors contributed equally \\ to this work. \\ *Correspondence to: \\ Kim JG, \\ Tel: +82-42-865-3961 \\ Fax: +82-42-865-3939 \\ E-mail: jjintta@kbsi.re.kr \\ Yoon $\mathrm{KB}$ \\ Tel: +82-2-715-2569 \\ Fax: +82-2-706-4269 \\ E-mail: yoonkb@sogang.ac.kr
}

Received October 28, 2015

Revised November 23, 2015

Accepted November 23, 2015

\begin{abstract}
Sample preparation is very important for crystal structure analysis of novel nanostructured materials in electron microscopy. Generally, a grid dispersion method has been used as transmission electron microscope (TEM) sampling method of nano-powder samples. However, it is difficult to obtain the cross-sectional information for the tabularstructured materials. In order to solve this problem, we have attempted a new sample preparation method using focused ion beam. Base on this approach, it was possible to successfully obtain the electron diffraction patterns and high-resolution TEM images of the cross-section of tabular structure. Finally, we were able to obtain three-dimensional crystallographic information of novel zeolite nano-crystal of the tabular morphology by applying the new sample preparation technique.
\end{abstract}

Key Words: Electron crystallography, Tabular structure, Sample preparation, Grid dispersion, Focused ion beam

\section{INTRODUCTION}

In the past few decades, hundreds of novel nanostructured materials (NSMs) exhibiting extraordinary properties compared with their bulk counterparts have been synthesized by a variety of methods with controlling the geometrical factors (Jun et al., 2005). To characterize the NSMs, extensive research has been conducted with transmission electron microscope (TEM) which is an indispensable tool for analyzing the novel physical and chemical properties of individual materials on the atomic scale (Neumann et al., 2010). In the characterization of the NSMs with the TEM, the most common method for TEM specimen preparation is the dispersion of the NSMs diluted in solution (commonly ethanol or water) as thinly as possible on the amorphous thin carbon film coated upon several metal grids.

Even if the steps for the method are not complicated and difficult to follow, some specific shapes of one or two dimensional materials such as belt, disk and plate, are mostly existed to be observed in one direction in the TEM analysis. In this case, it is hard to collect the crystal information of other directions, even though crystal structures obtained at least in three major directions are essential to solve the unknown crystal structure of NSMs (Sun et al., 2010). Moreover, NSMs with complex crystal structures have been produced through the newly developed synthesis process (Chen et al., 2011; Tadjarodi et al., 2013). Therefore, it has been strongly demanded to find out the sample preparation method to

This work was supported by Korea Basic Science Institute grant (D35612) to J. -G. Kim.

@ This is an open-access article distributed under the terms of the Creative Commons Attribution Non-Commercial License (http://creativecommons.org/licenses/by-nc/4.0) which permits unrestricted noncommercial use, distribution, and reproduction in any medium, provided the original work is properly cited.

Copyrights @ 2015 by Korean Society of Microscopy 
get the crystal information in the specific site of NSMs. In this study, we tried to overcome the weakness with advanced focused ion beam (FIB) techniques. One of the synthetic zeolites was chosen to be applied for the techniques because they have mostly a complex crystal structure (Corma, 1995; $\mathrm{Na}$ et al., 2011). Finally, we reported a new method to get the crystal information in the specific site in any kind of shapes of NSMs.

\section{MATERIALS AND METHODS}

To apply and test an advanced FIB sampling method, the hexagonal tabular morphology of the zeolite, has been synthesized under mild hydrothermal condition. Two kinds of sample preparation methods for TEM analysis were applied. Firstly, the grid dispersion was conducted to analyze the size, shape and electron diffraction (ED) pattern of the sample in [100] direction. Secondly, a cross-section TEM sample was prepared by a FIB (Quanta 3D FEG; FEI Co., USA). In the case of the grid dispersion method, the nanopowders were diluted with ethanol (20 v\%, nano-powder). After sonication for 20 minutes to minimize aggregation, the solution was released on the lacey carbon supported film of 300 mesh Cu grid (TED PELLA lnc., USA) and completely dried at room temperature. In the case of the FIB method, on the other hand, $\mathrm{Ga}^{+}$ion milling was conducted with gradually lowering ion beam current from 7 to $0.1 \mathrm{nA}$ to minimize the ion beam damage. Energy-filtered TEM (120 kV, LEO912 OMEGA; Carl Zeiss, Germany) was used to observe crystallite sizes, shapes and ED patterns of the zeolite nano-crystals. Additionally, high-resolution TEM (HRTEM) images and ED patterns of main zone axes were acquired by using an aberration corrected energy-filtered TEM (200 kV, Libra200 HT Mc Cs TEM; Carl Zeiss). ED patterns with dynamical effects reduced through an electron beam precession unit (SpnnigStar P020; NanoMEGAS, Belgium). The data analysis of the ED patterns and HRTEM images were performed using program CRISP \& ELD (Calidris, Sweden).

\section{RESULTS AND DISCUSSION}

Through the grid dispersion method, it was found that the zeolite nano-crystals had a hexagonal tabular morphology. Their average size and thickness were about $600 \mathrm{~nm}$ and 90 $\mathrm{nm}$, respectively. As shown in Fig. 1, it could be obtained easily the planar-directional ED pattern. This zone axis was determined discretionally to be the [100] direction through ED analysis (Fig. 2D). However, it is difficult to obtain ED patterns of other orthogonal direction of the tabular structure by the grid dispersion method because of its dispersion characteristic and thickness problem.

To complement these drawbacks of the grid dispersion method, we tried to produce a cross-section of zeolite nanocrystal using FIB. It was conducted by pre-treatment process in three different ways. At first, the zeolite nano powder was dispersed in ethanol solution and dropt on a silicon substrate and then C-coating was carried out with a thickness of approximately $100 \mathrm{~nm}$ as shown in Fig. 3A. After dispersing the sample on glass substrate, secondly, C-coating was performed in the same thickness of the first (Fig. 3B). Thirdly, samples were dispersed on the C-coated glass substrate and then additional $\mathrm{C}$-coating was performed like sandwich packing (Fig. 3C).

Samples prepared in the pre-treatment process of the three different methods were milled by FIB in the direction of [010] and [001]. At this time, we selected a sample having a complete hexagonal tabular morphology. Also, the milling directions were determined by the consideration of crystallographic orientation of ED pattern obtained from the planar-directional ED patterns ([100] zone axis).

In the first way, the silicon substrate is often used as FIB sampling material because it is a single crystal and has excellent thermal conductivity (Ferrando-Villalba et al., 2014). However, a disadvantage was found that the peak overlap (red circles) occurred in ED patterns obtained from both silicon substrate and zeolite nano-crystal as shown in Fig. 4A. Furthermore, it was difficult to make the zone axis ED patterns of zeolite nano-crystal due to the relatively strong excitation peaks diffracted from Si substrate.

In order to solve this problem, we used an amorphous glass substrate instead of a silicon substrate. In the case of the glass substrate, there was no peak overlap but zeolite nano-crystal was amorphized as shown in Fig. 4B. This amorphization phenomenon could be estimated from previous report (Park

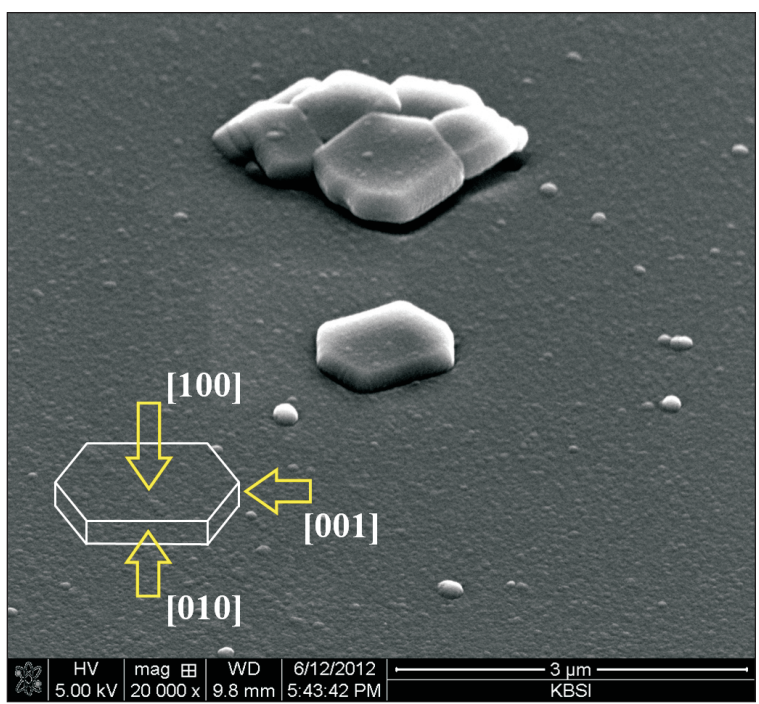

Fig. 1. The scanning electron microscope image shows that the zeolite has a hexagonal tabular morphology. 

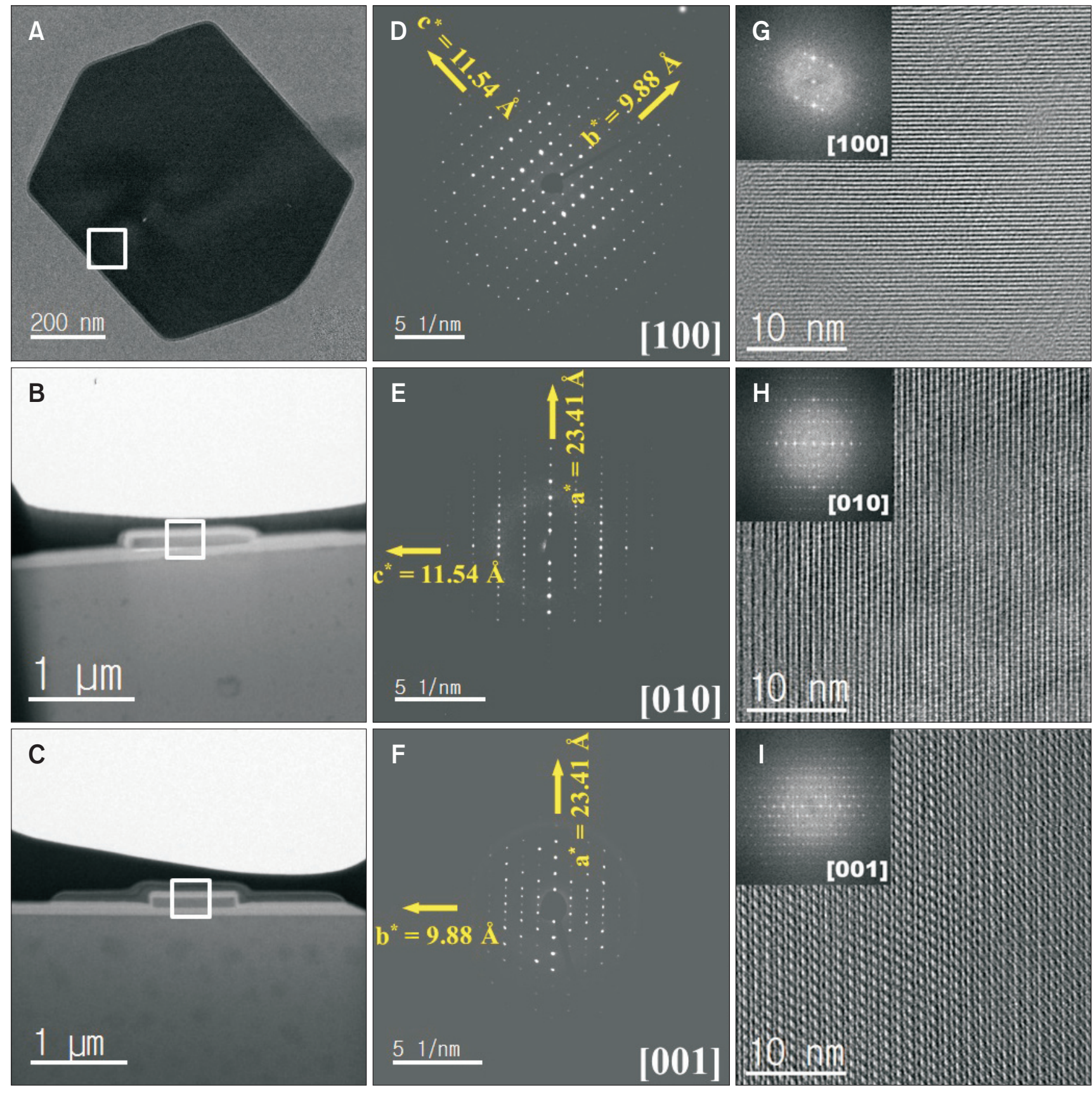

Fig. 2. The three major zone-axes diffraction patterns and high-resolution transmission electron microscope (HRTEM) images obtained from zeolite nanocrystal; the bright-field TEM images of two-dimensional tabular and cross sectional zeolite nano-crystal, respectively (A-C), electron diffraction patterns (D-F), HRTEM images (G-I).

et al., 2007). According to previous report, an amorphous glass endured the annealing effect of about $330^{\circ} \mathrm{C}$ during the $\mathrm{Ar}^{+}$ion milling. Moreover, $\mathrm{Ga}^{+}$ions in FIB system are accelerated with higher energy than $\mathrm{Ar}^{+}$ions. In this manner, zeolite nano-crystals seem to be damaged by heat conduction from the glass substrate during $\mathrm{Ga}^{+}$ion milling in FIB system. The third method was subjected to C-coating on a glass substrate to prevent the thermal conduction from glass substrate to zeolite nano-crystal. In this attempt, it was possible to obtain clear ED pattern for the orthogonal direction of zeolite nano-crystal without the peak overlap and thermal damage as shown in Fig. 4C.

As a result, we could obtain three-dimensional crystallographic information of zeolite nano-crystal as shown in Fig 2. 

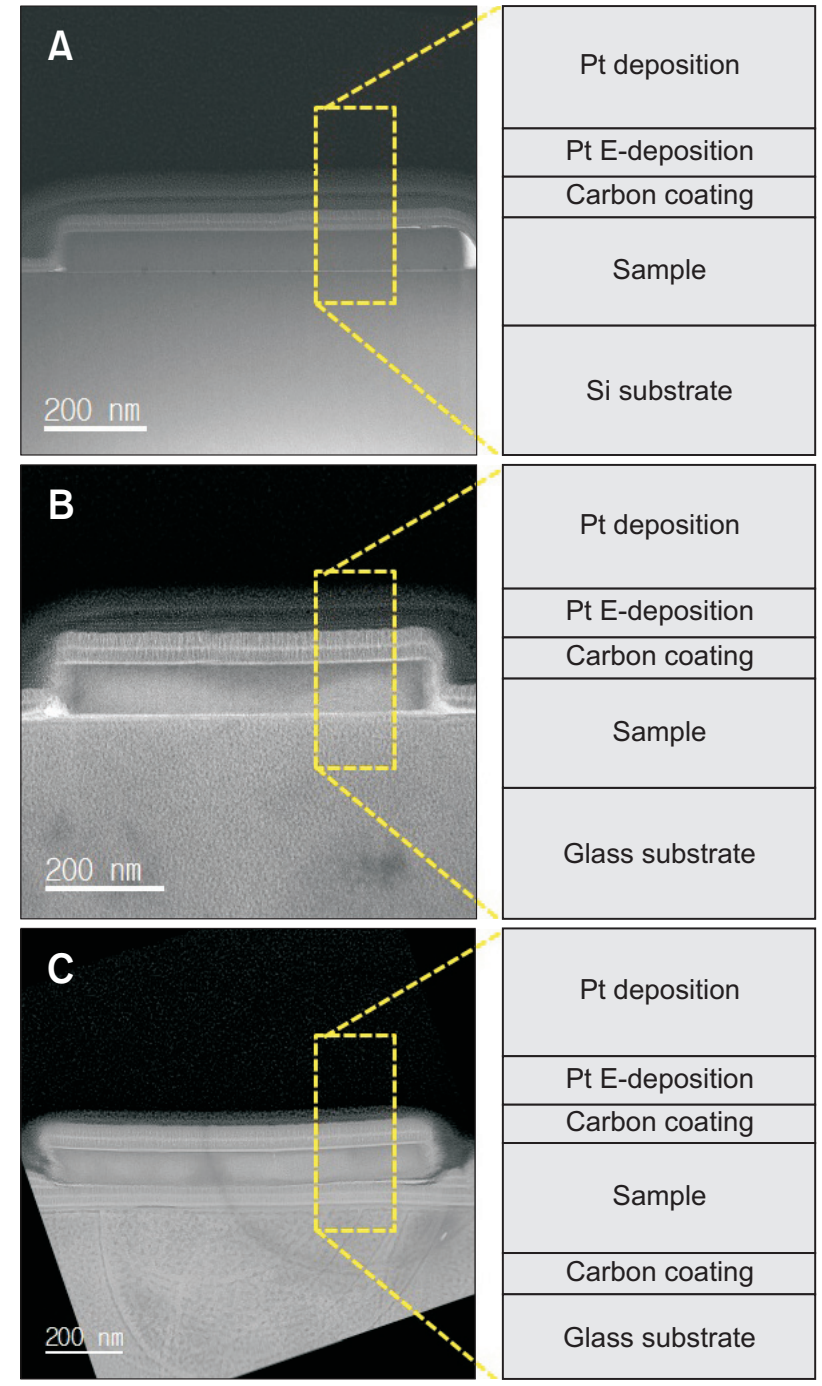

Fig. 3. The focused ion beam sampling methods; conventional deposition \& cross-section of the zeolite nano-crystal on a silicon substrate (A), on an amorphous glass substrate (B), and advanced preparation by applying the double $\mathrm{C}$-coating of the zeolite on an amorphous glass substrate (C).
Fig. 2A-C indicate the bright-field TEM images of three major orthogonal directions. The ED patterns of three major zone axes of zeolite nano-crystal were also obtained as shown in Fig. 2D-F. Each ED patterns was obtained by using precession ED technique. Therefore, all ED patterns have reliable peak intensities for structure solving even though their sample thickness are somewhat thick (about $90 \mathrm{~nm}$ ) (Kim et al., 2010). In addition, we obtained successfully HRTEM images of three major zone axes (Fig. 2G-I). Finally, it was revealed that the crystal structure of zeolite nano-crystal was orthorhombic lattice system with unit cell parameters which have $\mathrm{a}=23.41 \AA$, $b=9.88 \AA, c=11.54 \AA, \alpha=90^{\circ}, \beta=90^{\circ}, \gamma=90^{\circ}$. Also it is expected that its atomic structure could be solved with combination of ED patterns and HRTEM images.

\section{CONCLUSIONS}

In this study, we found and demonstrated the advanced FIB sampling method for three-dimensional crystal structure analysis of two dimensional zeolite nano-crystal. As it is expected, FIB is a very useful equipment for the site specific cross-section TEM specimen preparation of tabular structure with the preferred orientation compared to other sampling methods, such as grid dispersion or ultramicrotoming methods. Especially, we could determine the crystal structure of tabular zeolite nano-crystal through the successful extraction of crystallographic information with double C-coating FIB technique. Therefore, this FIB sampling method is expected to be very useful in structure analysis of various two dimensional soft-materials

\section{CONFLICT OF INTEREST}

No potential conflict of interest relevant to this article was reported.
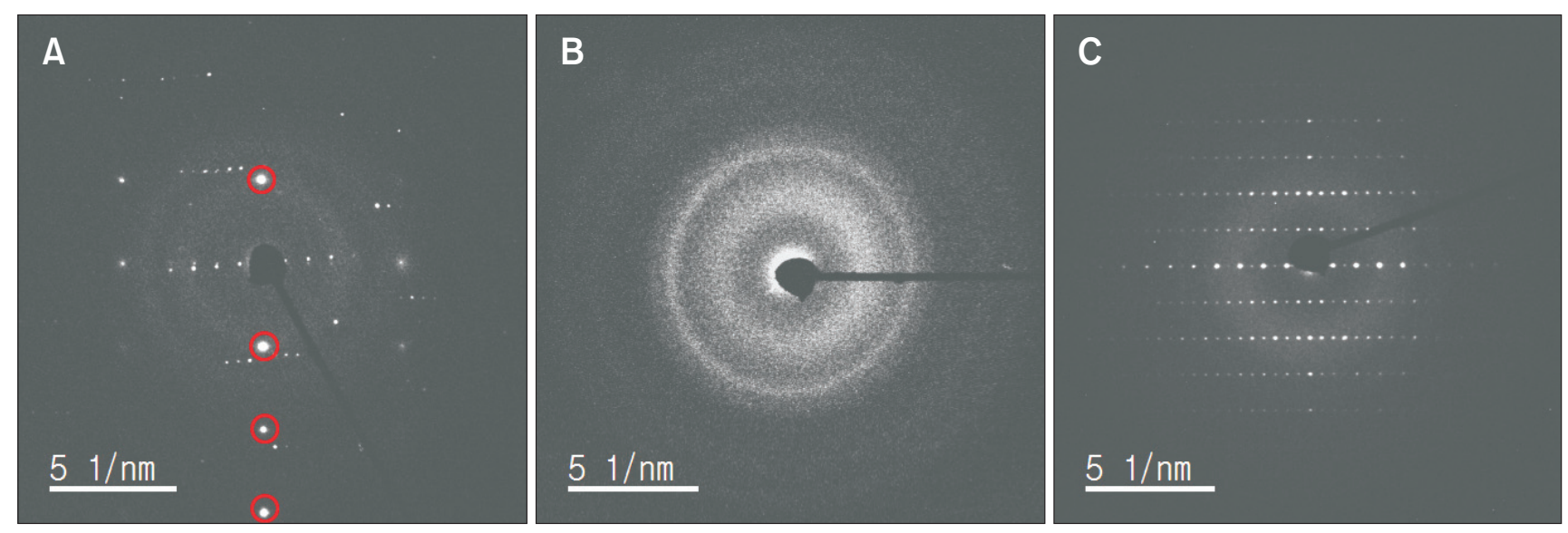

Fig. 4. The electron diffraction (ED) patterns obtained by three sample preparation processes using FIB; the ED pattern overlapped by both diffraction of silicon substrate and zeolite (A), the amorphous ED pattern due to thermal damages (B), and the perfect orthogonal ED pattern (C). 


\section{REFERENCES}

Chen Y, Xu Z, Gartia M R, Whitlock D, Lian Y, and Liu G L (2011) Ultrahigh throughput silicon nanomanufacturing by simultaneous reactive ion synthesis and etching. ACS Nano 5, 8002-8012.

Corma A (1995) Inorganic solid acids and their use in acid-catalyzed hydrocarbon reactions. Chem. Rev. 95, 559-614.

Ferrando-Villalba P, Lopeandia A F, Abad L I, Llobet J, Molina-Ruiz M, Garcia G, Gerbolès M, Alvarez F X, Goñi A R, Muñoz-Pascual F J, and Rodríguez-Viejo J (2014) In-plane thermal conductivity of sub-20 $\mathrm{nm}$ thick suspended mono-crystalline Si layers. Nanotechnology 25, 185402.

Jun Y W, Lee J H, Choi J S, and Cheon J W (2005) Symmetry-controlled colloidal nanocrystals: nonhydrolytic chemical synthesis and shape determining parameters. J. Phys. Chem. B. 109, 14795-14806.

Kim J G, Song K, Kwon K, Hong K, and Kim Y J (2010) Structure analysis of inorganic crystals by energy-filtered precession electron diffraction. J. Electron Microsc. 59, 273-283.
Na K, Jo C, Kim J, Cho K, Jung J, Seo Y, Messinger R J, Chmelka B F, and Ryoo R (2011) Directing zeolite structures into hierarchically nanoporous architectures. Science 333, 328-332.

Neumann W, Kirmase H, Hausler I, Mogilatenko A, Zheng $\mathrm{CH}$, and Heraba W (2010) Advanced microstructure diagnostics and interface analysis of modern materials by high-resolution analytical transmission electron microscopy. Bull. Pol. Ac.: Tech. 58, 237-253.

Park Y M, Ko D S, Yi K W, Petrov I, and Kim Y W (2007) Measurement and estimation of temperature rise in TEM sample during ion milling. Ultramicroscopy 107, 663-668.

Sun J, He Z, Hovmöller S, Zou X, Gramm F, Baerlocher C, and McCusker L B (2010) Structure determination of the zeolite IM-5 using electron crystallography. Z. Kristallogr. 225, 77-85.

Tadjarodi A, Izadi M, and Imani M (2013) Synthesis and characterization of the special ZnO nanostructure by mechanochemical process. Mater. Lett. 92, 108-110. 